\title{
Methods of Experimental Investigation of Cavitation in a Convergent - Divergent Nozzle of Rectangular Cross Section
}

\author{
Jablonská Jana $^{1}$, Kozubková Milada ${ }^{1}$, Himr Daniel ${ }^{2}$, Weisz Michal ${ }^{1}$ \\ ${ }^{1}$ Faculty of Mechanical Engineering, VŠB - Technical University of Ostrava 17. listopadu 15, 70833 Ostrava - Poruba, \\ Czech Republic, jana.jablonska@vsb.cz, milada.kozubkova@vsb.cz,michal.weisz@vsb.cz \\ ${ }^{2}$ VUT - Brno University of Technology, Technická 2896/2, 61669 Brno, Czech Republic, email: himr@fme.vutbr.cz
}

\begin{abstract}
Cavitation is a phenomenon with both positive and negative effects and with dynamic manifestations in hydraulic, food, chemical and other machinery. This article deals with the detection and dynamic behavior of cavitation clouds in water flows through a rectangular cross-section convergent-divergent nozzle. Cavitation was measured by methods applicable in engineering practice. Pressure, flow rate, noise, vibration, and amount of air dissolved in the liquid were measured and cavitation region was recorded with a high-speed camera. Evaluation of acquired images in connection with measured pressure pulsations and mechanical vibrations was performed with the use of the FFT method. In certain cases, dimensionless parameters were used to generalize the measurements. The results will be used to specify multiphase mathematical cavitation model parameters.
\end{abstract}

Keywords: Cavitation, diagnostics, experiment, dimensionless analysis, evaluation.

\section{INTRODUCTION}

Cavitation is a well-known, but still imperfectly described phenomenon characterized by random development and disappearance of vapor and gas bubbles under specific conditions. Cavitation can be, therefore, gaseous (released dissolved gas) or vaporous (liquid water turns into water vapor). This phenomenon occurs in liquids in areas where the pressure of the liquid drops to a low enough value. Reincreasing pressure causes the water vapor to condense, but the released air needs certain time to re-dissolve. The amount of air that can be dissolved in water is described by Henry's law; the rate of dissolution is described by Fick's law. It is practically impossible to differentiate between vaporous and gaseous cavitation in experimental observations and both types must be seen as one cavitation cloud [1].

Dynamic pressure drop when liquid passes through a constriction (e.g., in a convergent-divergent nozzle) is the most common cause of cavitation in hydraulic machinery. This phenomenon is referred to as hydrodynamic cavitation. In hydraulic practice, it usually indicates insufficient suction pressure. Cavitation can be very destructive to internal parts of pumps. In particular, it can cause erosion of impeller blades. Its presence is often manifested by noise as if gravel passes through the pump [2].

There are many situations when cavitation occurs. During water hammer, pressure drops significantly and cavitation may occur in low static pressure systems reinforcing the adverse effects of the water hammer. Autrique [3] did extensive research on the subject. An acoustic signal can also cause cavitation. Acoustic cavitation is utilized, for example, in sonochemistry [4], [5]. Around profiles (ship propellers, turbine blades, etc.), the flow can separate and cavitation may occur causing noise, vibration and erosion of machinery [6], [7]. Cavitation due to an abrupt enlargement of the flow cross-section can be classified in a similar category, as the flow separates too in this case [8]. Flow separation can be used in the production of nanopowders [9] or for material disgregation [10]. In unstable flow areas (draft tubes in turbines), a helical vortex (vortex rope) is formed, being a potent source of pressure pulsations and making the operation of Francis turbines impossible [11]. In flows through globe valves, static pressure drops and cavitation may occur [12]. Particularly interesting is the use of cavitation for destruction of cyanobacteria [13]. Generally, the occurrence of cavitation is determined by flow conditions and area geometry.

This work is focused on the observation of cavitation in a rectangular cross-section convergent-divergent nozzle with the aim to identify the cavitation cloud characteristics and to obtain parameters for mathematical models of multiphase cavitating flows.

The methods used to identify cavitation presence were [14]:

- Measurements of pressure, flow rate, noise, and oxygen content in the liquid - the gas present in the liquid 
changes its volume substantially upon pressure change. The volume changes generate noise; corresponding pressure pulsations are different in amplitude and frequency from those appearing in non-cavitating flows [15].

- Vibration measurement - the presence of cavitation in the system causes vibration of the system at frequencies of approximately $10^{3} \mathrm{~Hz}$.

- High-speed camera recording - acquired images are processed using special mathematical algorithms and the distribution of gaseous and liquid phases in the flow is analyzed.

Other methods to observe cavitation include [14], [16]:

- Light sensing - the collapse of cavitation bubbles is associated with local increase in temperature and luminescence of the liquid.

- PIV (Particle Image Velocimetry) - is an optical, flow visualization method used to find instantaneous distribution of velocities in a flow field. Theoretically, the method requires that particles be present in the liquid; velocity is calculated on the basis of their motion. In practice, the particles are represented by impurities naturally present in the liquid. Particles in the liquid may deform the cavitation region.

- Bubble spectrometer - is an instrument to determine the distribution of bubbles according to their diameter.

- Tomographic method - is based on capacitive or resistive principle, which allows observation of spatial cavitation regions in both water and oil (bubbles of large diameters).

Some methods strictly require the area with cavitation to be visible; therefore, they cannot be used in practical applications.

\section{EXPERIMENTAL APPARATUS}

A rectangular cross-section convergent-divergent nozzle made of transparent material was the principal part of the experimental apparatus used for the generation and examination of the cavitation cloud.

As shown in Fig.1., the experimental apparatus consisted of a vertical multistage centrifugal pump (nominal flow rate $18.4 \mathrm{~m}^{3} / \mathrm{h}=5.11 \cdot 10^{-3} \mathrm{~m}^{3} / \mathrm{s}$; head $62.6 \mathrm{~m}$ ) connected to a tank. The pump was powered by an electric motor with speed (and thus flow rate) controlled by YASKAWA VS mini J7 frequency inverter. Next, a flow meter (flow rate measurement range $(0.72-72) \mathrm{m}^{3} / \mathrm{h}$, i.e. $\left(2 \cdot 10^{-4}-2 \cdot 10^{-2}\right)$ $\mathrm{m}^{3} / \mathrm{s}$; accuracy $0.5 \%$ ) and the measured object - a transparent rectangular cross-section convergent-divergent nozzle (see Fig.1. for its dimensions) - were connected in the circuit. Upstream and downstream of the nozzle, Hydrotechnik pressure sensors with sampling frequency $0.001 \mathrm{~s}$ (measurement range $(-1-6) \mathrm{bar}$, i.e. (-0.1 $0.6) \mathrm{MPa}$; accuracy $1 \%$ ) to create $10 \mathrm{~s}$ time records of measured pressures with scanning intervals of $1 \mathrm{~ms}$ were used. To calculate the steady state, the measured data were averaged. At the moment when cavitation appeared in the circuit, recording of images captured by the Baumer HXC20c high-speed camera with the frequency of
280 frames per second started and was downloaded into the PC. The cavitation cloud length was then evaluated from the record. For investigated flow rates, sound, vibration, and dissolved oxygen values were recorded simultaneously. Individual measurements were then evaluated. The measurement range was limited by structural and material parameters of the equipment.

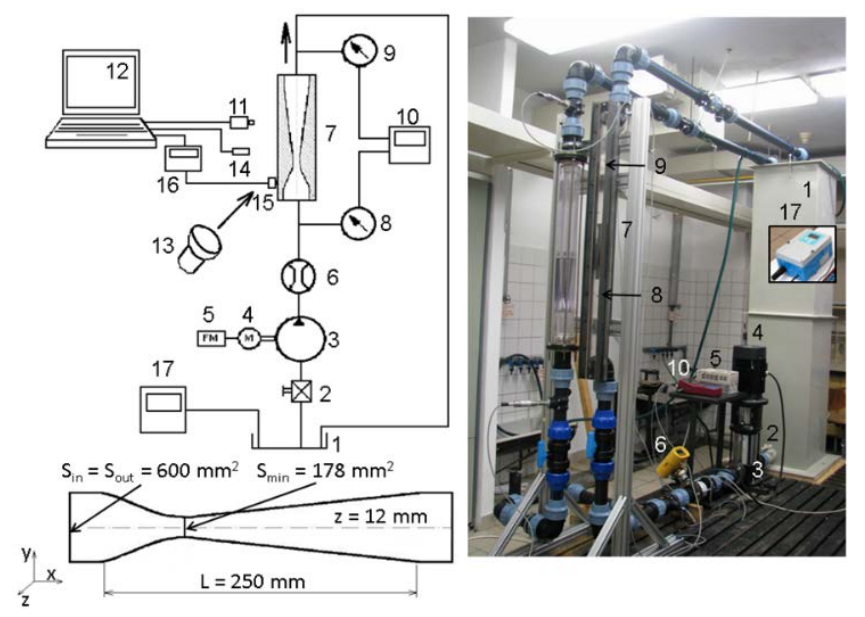

Fig.1. Measuring circuit diagram and basic parameters of the investigated object 1 - tank, 2 - ball valve, 3 - GRUNDFOS fivestage water pump, 4 - electric motor, 5 - YASKAWA VS mini J7 frequency inverter, 6 - ELIS PLZEN electromagnetic flow meter, 7 - measured object - rectangular cross-section nozzle, 8, 9 Hydrotechnik pressure sensors, 10 - Hydrotechnik analyzer, 11 Baumer HXC20c high-speed camera with CMOSIS CMV2000 sensor, 12 - PC with analysis software, 13 - light source, 14 - 1/2“" TYPE 489 microphones, 15 - vibration sensor, 16 - Microlog analyzer, 17 - ENDRESS + HAUSER LIQUISYS-M dissolved oxygen transmitter.

The diffuser was designed so that the enlargement is less than $5^{\circ}$, thus ensuring that separation of water flow will not occur. [23] The nozzle was placed in a vertical position in order to obtain symmetrical cavitation area (elimination of gravitational force influence). Hydrostatic pressure due to the distance of pressure sampling points (Fig.1. position 8 and 9) is negligible.

Measurements were performed at various flow rates in the range $2.62 \cdot 10^{-3} \mathrm{~m}^{3} / \mathrm{s}-4.6 \cdot 10^{-3} \mathrm{~m}^{3} / \mathrm{s}$ (corresponding to the frequency range between $20 \mathrm{~Hz}$ and $38 \mathrm{~Hz}$ of the frequency inverter to control the pump) at which cavitation formed. The maximum flow rate reached $4.36 \cdot 10^{-3} \mathrm{~m}^{3} / \mathrm{s}$; at this flow rate, the velocity of the liquid in the $0.05 \mathrm{~m}$ diameter pipe was $2.2 \mathrm{~m} / \mathrm{s}$, the velocity at the rectangular cross-section inlet ( $\mathrm{S}=600 \mathrm{~mm}^{2}$ ) was $7.3 \mathrm{~m} / \mathrm{s}$ and the velocity at the narrowest point of the nozzle $\left(\mathrm{S}=178 \mathrm{~mm}^{2}\right.$ ) was $24.5 \mathrm{~m} / \mathrm{s}$. The relative pressure at the inlet to the measured area was $226624 \mathrm{~Pa}$, the relative pressure at the outlet from the measured area was $81261 \mathrm{~Pa}$ and the cavitation region length was $95 \mathrm{~mm}$.

The water temperature was constant $\left(20^{\circ} \mathrm{C}\right)$, because the reservoir had a volume of $500 \mathrm{l}$ and each measurement was carried out for 10 seconds. Therefore, water did not circulate 
in the system for a longer time. This prevented its heating which was obvious from temperature measurement. The saturation vapor pressure of water at $20^{\circ} \mathrm{C}$ is approximately $2340 \mathrm{~Pa}$.

The measurements were executed repeatedly within several weeks to demonstrate repeatability and reproducibility of the measurement results. Deviation of the result values in repeated measurements was $\pm 2 \%$ for pressure and $\pm 2 \%$ for flow rate.

Table 1. Presented variants.

\begin{tabular}{|c|c|l|}
\hline variant & flow rate & \multicolumn{1}{c|}{ status } \\
\hline A & $2.52 \cdot 10^{-3} \mathrm{~m}^{3} / \mathrm{s}$ & no cavitation \\
\hline B & $3.38 \cdot 10^{-3} \mathrm{~m}^{3} / \mathrm{s}$ & cavitation cloud initiation \\
\hline C & $3.9 \cdot 10^{-3} \mathrm{~m}^{3} / \mathrm{s}$ & cavitation \\
\hline D & $4.36 \cdot 10^{-3} \mathrm{~m}^{3} / \mathrm{s}$ & $\begin{array}{l}\text { cavitation at maximum flow } \\
\text { rate }\end{array}$ \\
\hline
\end{tabular}

\section{METHODS - DIMENSIONLESS PARAMETERS}

\section{Cavitation number}

Cavitation number depends on the geometry of the region where cavitation is expected to occur [14], [17], [18], so it can only be determined by measurement.

Cavitation at the narrowest point of the experimental nozzle is caused by increasing the flow rate in the hydraulic circuit above certain minimum value. As a result, an unsteady cavitation region is formed downstream of the narrowest point of the nozzle [2]. Generally, cavitation region size and therefore cavitation number depend on actual geometry, inlet or outlet pressure and maximum velocity at constant temperature [14].

$$
C N=C N\left(\rho, p, p_{n}, v\right)
$$

For experimental nozzle, cavitation number (dimensionless coefficient) can be defined as

$$
C N=\frac{p_{2}-p_{n}}{\rho \frac{v_{\max }^{2}}{2}}=\frac{2\left(p_{2}-p_{n}\right)}{\rho v_{\max }^{2}}
$$

where $p_{2}$ is the pressure at the outlet from the measured area [Pa] (Fig.1., position 9), $p_{n}$ is the vapor pressure of the liquid at the temperature of the experiment (at $20^{\circ} \mathrm{C} p_{n}=2340 \mathrm{~Pa}$ ), $\rho$ is the liquid density $\left[\mathrm{kg} \cdot \mathrm{m}^{-3}\right]$, and $v_{\max }$ is the liquid flow velocity at the narrowest point of the nozzle [m.s $\mathrm{s}^{-1}$.

The Reynolds number is a dimensionless parameter that specifies the flow conditions and relates inertial and friction forces (i.e., the resistance of the environment as a result of the internal friction). In general, it is defined as

$$
\operatorname{Re}=\operatorname{Re}\left(v, d_{h}, v\right)=\frac{v d_{h}}{v}
$$

where $d_{h}$ is hydraulic diameter [m], $v$ is the fluid flow mean velocity at given cross-section $\left[\mathrm{m}_{\mathrm{s}} \mathrm{s}^{-1}\right], v$ is the fluid kinematic viscosity $\left[\mathrm{m}^{2} \cdot \mathrm{s}^{-1}\right]$. For the experiment $d_{h}$ is the narrowing hydraulic diameter and $v$ is maximum velocity at the narrowed cross section of nozzle.

Equation (3), derived by substituting (2) into (1), describes the dependence of the cavitation number on the Reynolds number and the outlet pressure.

$$
C N=\frac{2\left(p_{2}-p_{n}\right)}{\rho v_{\max }^{2}}=\frac{2 d_{h}^{2}\left(p_{2}-p_{n}\right)}{\rho(\operatorname{Re} v)^{2}}
$$

\section{Strouhal number}

The cavitation region in a convergent-divergent nozzle is characterized by periodically formed bubbles entrained by flow. The bubbles never collapse at the place where they are formed (except vibratory cavitation) but they are carried downstream creating a cavitation cloud [14], [17].

When liquid flows through a nozzle, vortices form downstream of the nozzle. The vortex frequency is given by the Strouhal number (dimensionless coefficient)

$$
S t=\frac{f l_{k}}{v}
$$

where $f$ is the cavitation region frequency $[\mathrm{Hz}], l_{k}$ is the characteristic dimension [m] (length of cavitation field), $v$ is the mean flow velocity at the inlet to the measured area [m.s $\left.{ }^{-1}\right]$ (Fig.1., position 6). The Strouhal number is suitable for description of periodic events. According to Salnev [19], cavitation cloud formed in a diffuser is of a periodical character and the Strouhal number is in the range of $0.163-$ 0.179 , depending on the diffuser type. However, Hunsaker [20] found that the Strouhal number for diffusers lay in the range of $0.31-0.58$.

Dependence of the vortex frequency on the Strouhal number is defined

$$
f=\frac{v}{S t l_{k}}=f\left(S t, \operatorname{Re}, l_{k}, C N\right)
$$

\section{Determination of dimensionless parameters from experiment}

Formation of cavities was observed in the experiment. As flow rate was being increased, evaluation of images from the high-speed camera recording showed various cavitation region lengths. Cavitation started to emerge at the critical cavitation number of 0.98 being manifested by an intermittent noise; at the narrowest point of the nozzle (at the wall), two small cavitation clouds were appearing and disappearing due to the symmetry of the nozzle.

From the measurements, the basic characteristic of the hydraulic element was evaluated, i.e., $p$ - $Q$ characteristic. It was subsequently converted into dimensionless parameters, i.e. $\zeta$ - $R e$, see Fig.2. Loss coefficient is influenced by cavitation.

Fig.3. shows the dependence of cavitation parameters on the Reynolds number. It also shows coordinates of the critical point, i.e., the Reynolds number with the value 220000 when cavitation starts to form, and the cavitation number with the value of 0.98 . 


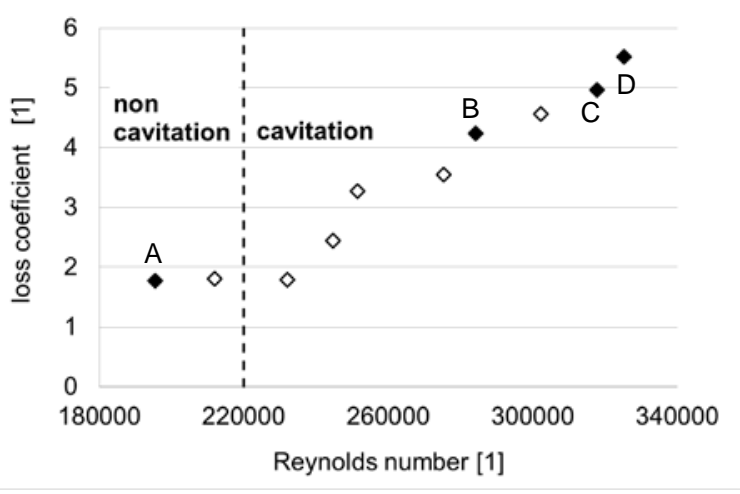

Fig.2. Dependence of the loss coefficient on the Reynolds number (experiment), filled marks show the measurements presented by A, B, C, D.

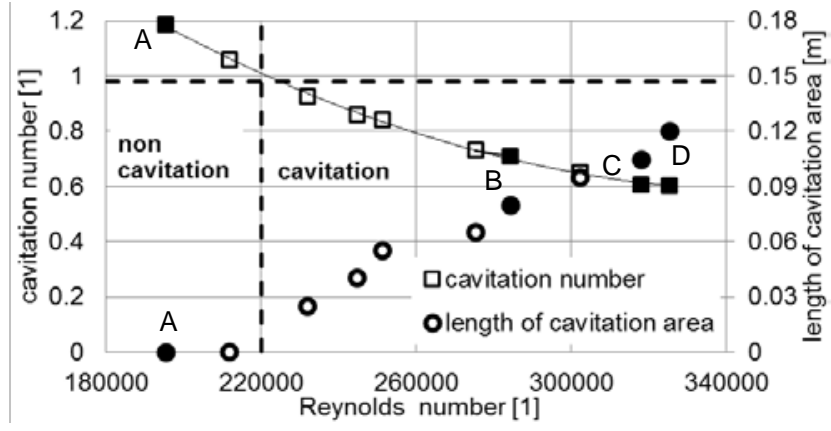

Fig.3. Dependence of the cavitation number and length of cavitation area on the Reynolds number (experiment), filled marks show the measurements presented by A, B, C, D.

In this experiment, the Strouhal number was determined in the range of $(0.12-0.22)$, which corresponds approximately with the observations of Salnev [19]. In Fig.4. the lines represent data $\mathrm{B}, \mathrm{C}, \mathrm{D}$, at which cavitation was detected.

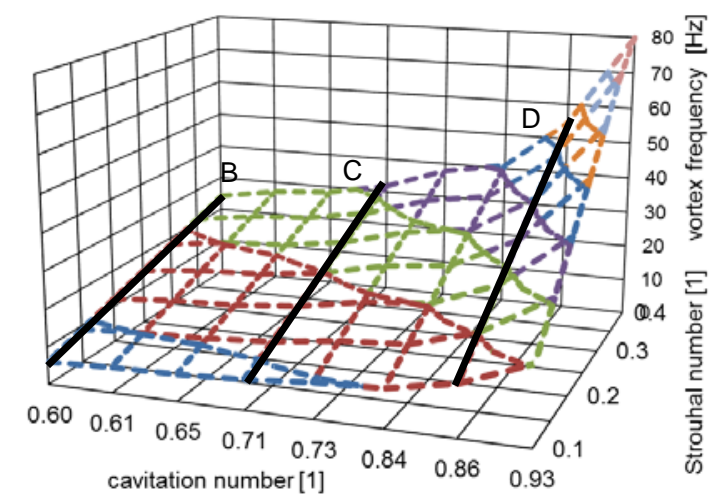

$=70-80=60-70=50-60=40-50 \approx 30-40=20-30=10-20=0-10$

Fig.4. Estimation of the vortex frequency vs. the cavitation number and the Strouhal number.

Fig.4. shows that the vortex frequencies lay in the range of $(10-60) \mathrm{Hz}$ at investigated pump flow rates. Therefore, further measurements will focus on this frequency range.
The experiment showed that:

- The flow was turbulent in all measurements.

- Cavitation started to occur at the flow rate of $0.003 \mathrm{~m}^{3} / \mathrm{s}$, i.e., at the Reynolds number of 220000.

- The critical cavitation number was 0.98.

- The cavitation cloud frequencies lay in the range of (10 60) $\mathrm{Hz}$.

\section{Methods - EVAluation OF THE MEASURED VALUES}

\subsection{Pressure and flow rate measurement}

A ruler was used for an indicative estimation of the cavitation region length; the exact measurement was carried out with the use of image analysis.

The time courses of pressure were evaluated for flow rates of $2.52 \cdot 10^{-3} \mathrm{~m}^{3} / \mathrm{s}$ (no cavitation), $3.38 \cdot 10^{-3} \mathrm{~m}^{3} / \mathrm{s}$ (cavitation cloud initiation), $3.9 \cdot 10^{-3} \mathrm{~m}^{3} / \mathrm{s}$, and $4.36 \cdot 10^{-3} \mathrm{~m}^{3} / \mathrm{s}$ (maximum flow rate). Pressure pulsations at investigated flow rates were subjected to the Fourier transform. At the flow rate of $2.52 \cdot 10^{-3} \mathrm{~m}^{3} / \mathrm{s}$, there was no cavitation yet. Fig.5. shows that at flow rates of $2.52 \cdot 10^{-3} \mathrm{~m}^{3} / \mathrm{s}$ and $3.38 \cdot 10^{-}$ ${ }^{3} \mathrm{~m}^{3} / \mathrm{s}$, no significant vortex frequency was apparent. At flow rates less than $3.38 \cdot 10^{-3} \mathrm{~m}^{3} / \mathrm{s}$, the cavitation region was almost stable. At maximum flow rate of $4.36 \cdot 10^{-3} \mathrm{~m}^{3} / \mathrm{s}$, a distinctive cavitation cloud frequency of $17 \mathrm{~Hz}$ was noticeable. This frequency was confirmed by other evaluation methods. The cavitation cloud frequency of $17 \mathrm{~Hz}$ was not present at other pump speeds because the cavitation region was considerably shorter; the pressure sensor was at a great distance from the cavitation region and so the pressure pulses were attenuated. The natural frequency of the system in the measurement was approximately $8 \mathrm{~Hz}$. In contrast to Fig.11., the pump rotational speed is not very apparent.

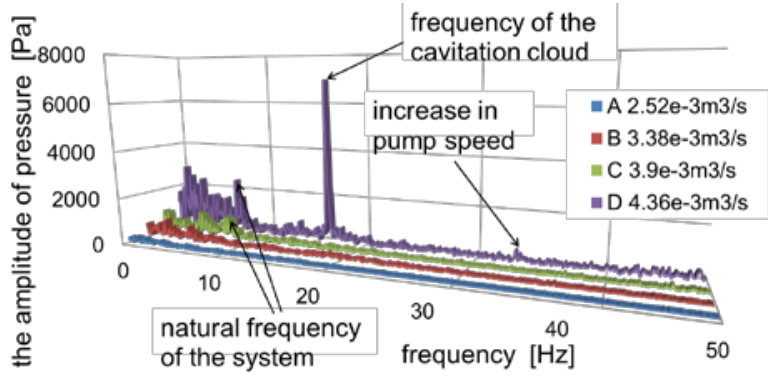

Fig.5. Frequency amplitude characteristics at investigated flow rates determined from the measured pressure.

\subsection{Noise measurement}

Sound pressure is the pressure deviation from the ambient pressure caused by a sound wave; it is measured in Pascal. The acoustic manifestation of cavitation bubbles is referred to as cavitation noise. Pearsall [21] showed that small cavitation bubbles emit noise with frequencies up to $3 \mathrm{MHz}$, whereas Sou [22] found that large bubbles could generate noise with frequencies up to $10 \mathrm{kHz}$. Cavitation noise increases from cavitation cloud initiation to the point when the cavitation is almost fully developed and then it decreases. The cavitation noise level may reach $90 \mathrm{~dB}$. 
Cavitation noise was measured with two Brüel \& Kjaer $1 / 2$ "TYPE 489 microphones perpendicular to each other and to the nozzle (see Fig.6.). The acquisition time was $30 \mathrm{~s}$ and the sampling frequency was $65565 \mathrm{~Hz}$. The acquired data from both sensors were analyzed by the Pulse Labshop software. The data could be converted to decibels using the expression $20 \cdot \log p / p_{0}$, where $p_{0}=2 \cdot 10^{-5} \mathrm{~Pa}$ is the reference sound pressure. The noise was measured in the laboratory; therefore, noise with frequencies lower than $30 \mathrm{~Hz}$ should be disregarded due to background noise. Fig.7. shows the frequency spectrum amplitude depending on frequency (logarithmic scale) obtained by the Fourier transform of the sound pressure. For frequencies to approximately $30 \mathrm{~Hz}$, the curves for investigated flow rates were affected by electronic noise. Therefore, it would be incorrect to take these frequencies into consideration. For frequencies above $100 \mathrm{~Hz}$, the sound pressure increased depending on flow rate $-2.52 \cdot 10^{-3} \mathrm{~m}^{3} / \mathrm{s}$ (no cavitation), $3.38 \cdot 10^{-3} \mathrm{~m}^{3} / \mathrm{s}$ (cavitation cloud initiation), $3.9 \cdot 10^{-3} \mathrm{~m}^{3} / \mathrm{s}$, and $4.36 \cdot 10^{-3}$ $\mathrm{m}^{3} / \mathrm{s}$ (maximum flow rate). The record showed that with increasing flow rate the sound pressure increased, i.e., the cavitation was louder. Full cavitation was apparent at frequencies greater than $500 \mathrm{~Hz}$.

Other system frequencies might be present in the record, e.g., at the maximum flow rate of $4.36 \cdot 10^{-3} \mathrm{~m}^{3} / \mathrm{s}$ there is a distinct peak at the frequency of $225 \mathrm{~Hz}$, which corresponds to the frequency of the five-stage centrifugal pump.

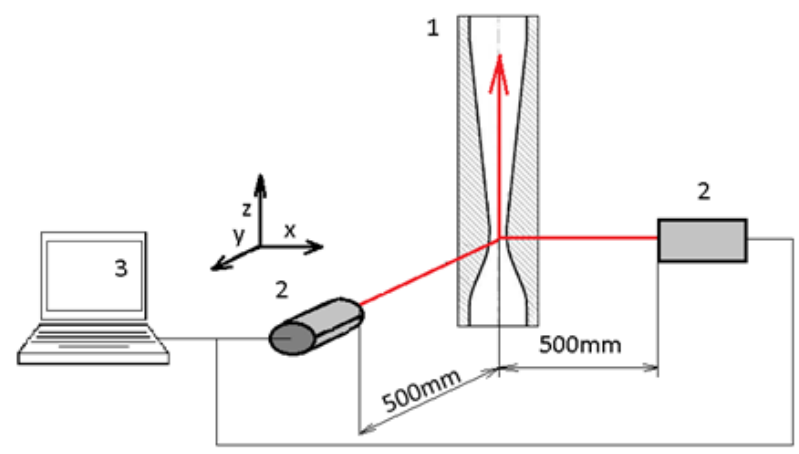

Fig.6. Noise measurement diagram: 1 - measured object rectangular cross-section nozzle, 2 - Brüel \& Kjaer microphones, 3 - PC with analysis software.

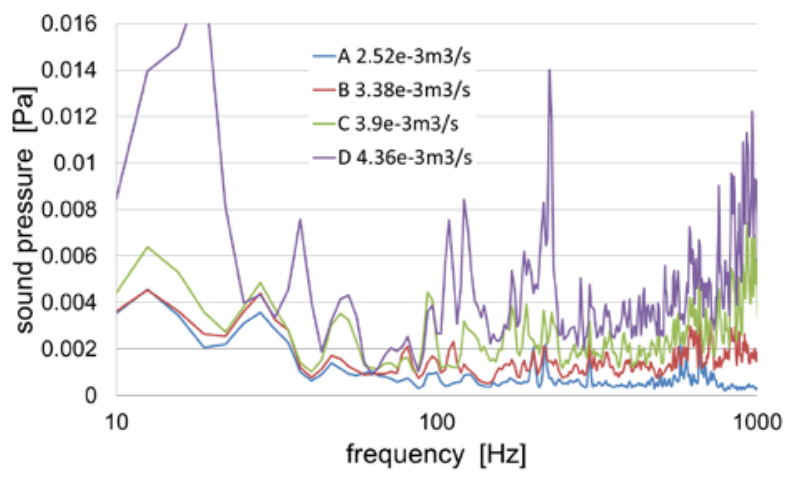

Fig.7. Frequency amplitude characteristics at investigated flow rates determined from the measured noise.

\subsection{Vibration measurement}

Vibration can be measured by various methods that provide greater or lesser amount of information. Overall vibration values measurement method is the simplest and most commonly used. The effective value of the vibration velocity in the ranges of $(10-1000) \mathrm{Hz}$ or $(2-1000) \mathrm{Hz}$ is usually measured according to ISO 10816.

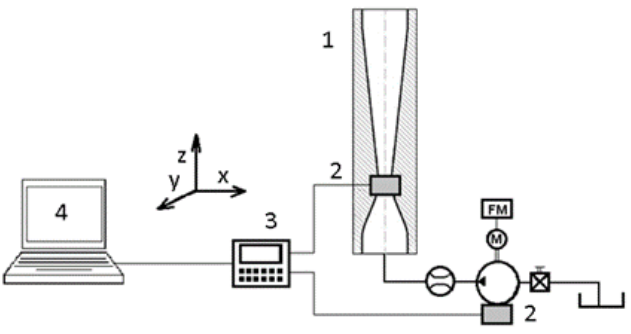

Fig.8. Vibration measurement diagram: 1 - measured object rectangular cross-section nozzle, 2 - CMSS 2200 accelerometer, 3 - Microlog CMXA 80 analyzer, 4 - PC with analysis software.

Vibration measurements were performed with the CMSS 2200 accelerometer (precision $0.5 \%$ ). Two accelerometers were placed in the hydraulic circuit - one on the nozzle and one on the pump (see Fig.8.). Vibration sensors were attached to the nozzle with a resinous adhesive and to the pump with a magnet. Data were collected in two channels to the Microlog CMXA 80 analyzer and then evaluated. Y-axis vibrations of the nozzle and vibrations of the pump were recorded for the investigated flow rates. X-axis vibrations of the nozzle were not significant as the nozzle was fixed to the frame and the frame to the floor.

In order to correctly identify the frequencies, it is important to identify the natural frequency of the structure (including the liquid at rest), for which a bump test is used. A structure at rest is hit with a hammer, which generates a vibration signal. The natural frequency of the system was identified with the bump test and its value was

$$
f_{\text {system }}=9 \mathrm{~Hz} \text {. }
$$

In the second measurement, performed as continuous, the pump flow rate was being increased gradually in steps of $2 \cdot 10^{-4} \mathrm{~m}^{3} / \mathrm{s}$ and vibration was recorded for 10 seconds at each flow rate. This measurement provided the most information as it covered the entire system and individual vibration sources were identifiable.

Cavitation could be detected particularly at higher frequencies (200 $\mathrm{Hz}$ and greater). Lower frequencies, clear in detailed record, correspond to the natural frequency of the system $(9 \mathrm{~Hz})$ and the frequency of the pump and the system (30 - $90 \mathrm{~Hz}$ range).

In next, more detailed measurement, the vibration sensor was placed on the nozzle and the pump flow was set to the investigated value. Vibration was recorded for 20 seconds and the record was subjected to FFT frequency analysis. The cavitation cloud frequency of $17 \mathrm{~Hz}$ is prominent. Unlike in pressure measurements, this frequency can also be observed at low flow rates because the vibration sensor was placed directly on the narrowest part of the rectangular crosssection convergent-divergent nozzle. 


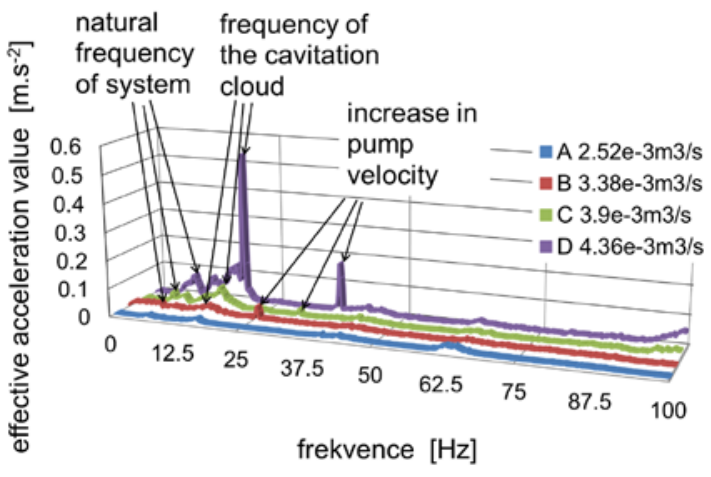

Fig.9. Frequency amplitude characteristics at investigated flow rates determined from the measured vibration.

Cavitation causes vibration of the system. Our aim was to observe the frequency of the cavitation cloud oscillation, not the frequency of a collapsing bubble. Not in all measurements the cavitation cloud oscillation frequency was clear. Its detectability increased with increasing flow rates and, thus, with increasing cavitation number and cavitation evolution. Other evaluation methods also showed that the vortex frequencies were more prominent at higher flow rates.

\subsection{Image analysis}

Camera recording was used to determine the frequencies of cavitation cloud pulsations. This approach promised the results would not be vitiated by errors due to interferences caused by other phenomena. When analyzing pressure records, for instance, it needs to be taken into account that some pulsations are not produced solely by flow separation in the nozzle, but also by vibrations of various system components, pump impeller rotation, or other causes.

Images with the pixel resolution of $(1500 \times 450)$ and pixel density of $8.6 \mathrm{px} / \mathrm{mm}$ were rendered in grayscale (see Fig.10.). This setting allowed image exposure with the frame rate of $280 \mathrm{fps}$. The flow cross section was rectangular; therefore, only two components of the flow velocity could be assumed to be dominant and the task could be solved as two-dimensional. The analysis proceeded as follows: The total number of pixels (which was proportional to the area in $\mathrm{mm}^{2}$ ) with the number defining the shade of gray greater than 0.169 (0 representing black and 1 representing white) was counted in all images. This simple approach proved to be sufficiently accurate. As a result of light refraction, an image distortion slightly increased with increasing distance from the nozzle throat, but the distortion was considered of minor significance and disregarded in image processing. The estimated size of the cavitation cloud area was $0.014 \mathrm{~mm}^{2}$. Also, the exposure time of $1 / 280 \mathrm{~s}$ for the area close to the axis passing through the nozzle throat proved to be too long as the flow was very fast there (up to $24.5 \mathrm{~m} . \mathrm{s}^{-1}$ ). As a result, the images were slightly blurred, but the blurs were not large enough to affect the analysis results.

The acquired time dependence of the cavitation cloud area was subjected to discrete Fourier transform. The result is shown in Fig.11. where, rather than one dominant frequency, several areas of higher amplitudes can be observed, especially at frequencies below $10 \mathrm{~Hz}$ and in the range of $(18-24) \mathrm{Hz}$. The results for frequencies above $28 \mathrm{~Hz}$ should not be considered reliable because of the relatively low frame rate of 280 fps (one cycle should be captured in ten frames at least).

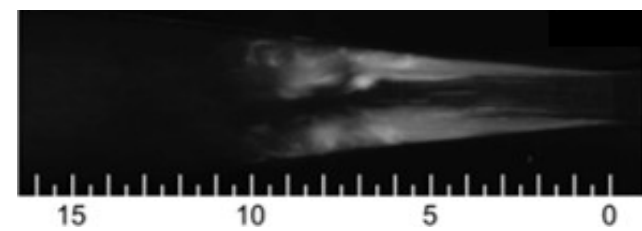

Fig.10. Cavitation at $4.36 \cdot 10^{-3} \mathrm{~m}^{3} / \mathrm{s}$. The scale $(0-16 \mathrm{~cm})$ is at the bottom of the picture. The flow direction is from right to left.

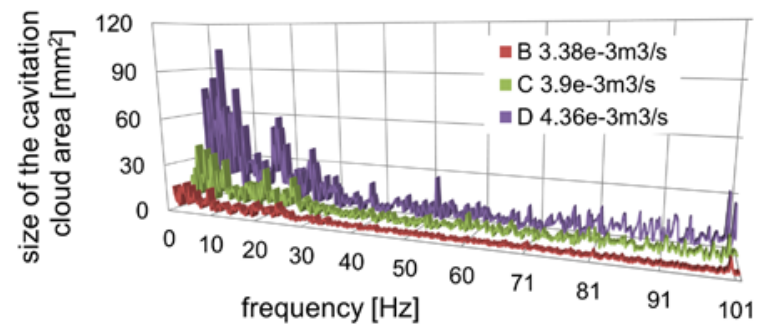

Fig.11. Frequency amplitude characteristics at investigated flow rates determined from the image analysis.

The average size of the cavitation region depending on the flow rate was also evaluated (see Fig.12.). The graph shows the dependence was parabolic from the minimum flow rate at which cavitation emerged.

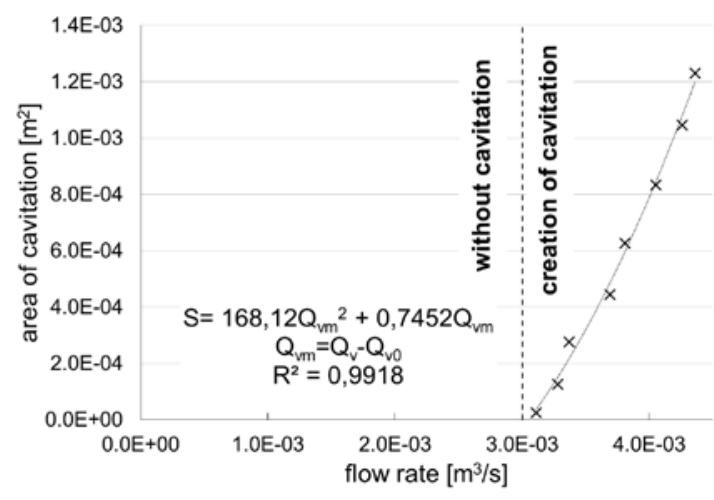

Fig.12. Average size of the cavitation region at different flow rates.

\section{Results}

The aim of this paper was to describe and evaluate different approaches to cavitation measurement and detection applicable in practice in diverse types of machinery.

In Fig.13., frequency analyses obtained from measurements are shown for investigated flow rates of $2.52 \cdot 10^{-3} \mathrm{~m}^{3} / \mathrm{s}$ (no cavitation), $3.38 \cdot 10^{-3} \mathrm{~m}^{3} / \mathrm{s}$ (cavitation cloud initiation), $3.90 \cdot 10^{-3} \mathrm{~m}^{3} / \mathrm{s}$, and $4.36 \cdot 10^{-3} \mathrm{~m}^{3} / \mathrm{s}$ (maximum flow rate). 

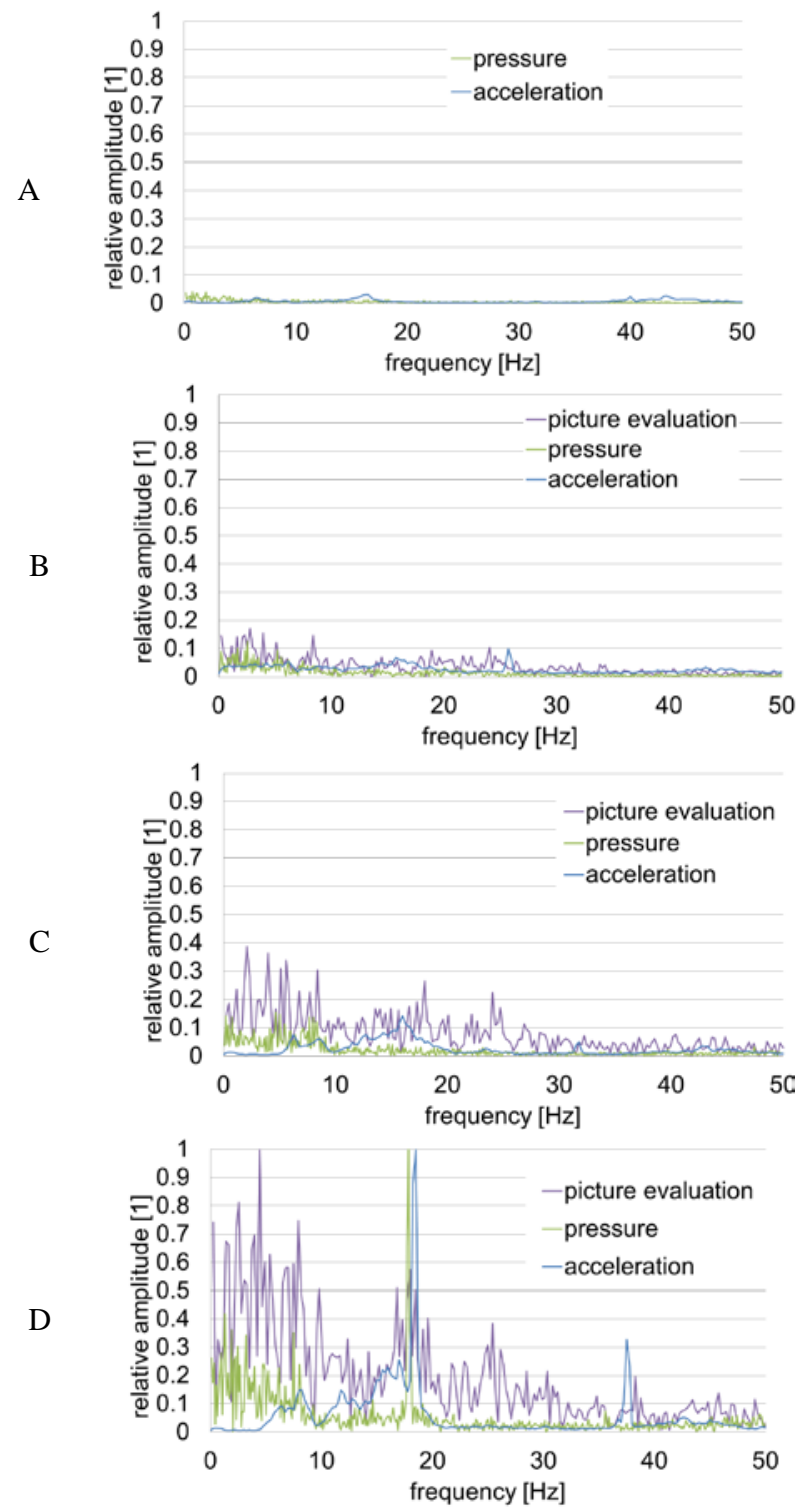

Fig.13. Frequency amplitude characteristics for investigated flow rates - the y-axis shows relative values in relation to maximum values for given flow rate.

The pressure measurement evaluation shows heavy interferences at frequencies below $10 \mathrm{~Hz}$ from pulsations generated in various points of the measuring circuit. The frequency characteristics as seen in Fig.13.A) show there is no cavitation forming in the nozzle. The initial phase of cavitation formation is characterized by frequencies with minimum amplitudes (see Fig.13.B)). The frequency characteristics of acceleration provide the most unambiguous results allowing identification of the causes of various frequencies (see Section 4.3.). The evaluation of frequency characteristics of image shows heavy interferences below $10 \mathrm{~Hz}$ for all flow rates. In Fig.13.D), frequency of approximately $18 \mathrm{~Hz}$ is apparent in all methods applied; it corresponds to the cavitation cloud frequency. In Fig.13.B) and Fig.13.C), significant frequencies are apparent; these are caused not by cavitation, but rather by pump operation, piping, etc.

\section{CONCLUSIONS}

Cavitation was examined in an experimental apparatus in a rectangular cross-section nozzle with transparent walls so that several methods of cavitation identification could be used. Cavitation was generated by flow separation from the walls in the divergent part of the nozzle. The size of the cavitation cloud and the frequency of the separation were investigated by measurements of pressure pulsations, vibration, noise, and by image analysis.

The measurement results were transformed to characteristic numbers (cavitation, Strouhal and Reynolds numbers) and they were found comparable to findings available in the literature dealing with the subject. The flow in the nozzle of rectangular cross-section was measured in the range of the cavitation number of $0.6-1.2$. The critical cavitation number was determined experimentally to be 0.98 . No cavitation formed when the cavitation number was greater than 0.98 . The critical cavitation number can only be determined by an experiment.

Pressure measurement downstream of the examined element is one of the methods suitable for cavitation identification. If it shows frequencies with minimum amplitudes (see Fig.13.B)), it is evident that initial phase of cavitation is formed in the element. This method is simple, fast, inexpensive and sufficient for preliminary identification of problems. Afterwards, the element should be examined more closely. The sound level of the noise identifies the approximate size of the bubbles - the lower the frequency, the larger the bubbles. Vibration measurement is the most exact method for cavitation identification in machinery. This method allows accurate detection of the cavitation cloud initiation and identification of the cavitation cloud oscillation frequency and other frequencies related to the hydraulic circuit and pump. Identification of cavitation with the use of images is hardly applicable in practice as most machinery elements are made of non-transparent materials. This method is, however, very important for accuracy improvement of mathematical models of multiphase cavitating flows.

The evaluation of image analysis and pressure and vibration measurements shows the cavitation cloud does not pulsate at one single frequency, but rather in a range of relatively low frequencies (less than $20 \mathrm{~Hz}$ ).

Air contained in water (dissolved and undissolved) has a great influence on the formation and size of the cavitation region. The undissolved air appears in the hydraulic circuit due to insufficient sealing of the circuit at places of pressure drop. More undissolved air is released from the air dissolved in the liquid; its content increases with increasing flow rate. All results and findings from frequency characteristics are particularly important for mathematical modeling. Mathematical models are used to predict the cavitation and, thus, it is easier to design high-quality hydraulic components. Mathematical models of multiphase cavitating flows need to be refined so that they can be used in practice. The mixture composition of the flowing medium (liquid, vapor, air) has particular influence on the hydraulic circuit parameters, the size of the cavitation region and the frequency of the pulsating cavitation cloud. 


\section{ACKNOWLEDGMENT}

The work presented in this paper was supported by a SGS grant SP2016/70.

\section{REFERENCES}

[1] Luo, J., Xu, W.L., Niu, Z.P., Luo, S.J., Zheng, Q.W. (2013). Experimental study of the interaction between the spark-induced cavitation bubble and the air bubble. Journal of Hydrodynamics, Ser. B, 25 (6), 895-902.

[2] Brennen, C.E. (2013). Cavitation and Bubble Dynamics. Cambridge University Press.

[3] Autrique, R., Rodal, E., Sánchez, A., Carmona, L. (2012) Physical model studies of water column separation. In IOP Conference Series: Earth Environmental Science, 15 (2012), 022014.

[4] Zhang, Y., Du, X., Xian, H., Wu, Y. (2015). Instability of interfaces of gas bubbles in liquids under acoustic excitation with dual frequency. Ultrasonics Sonochemistry, 23, 16-20.

[5] Lee, J., Kentish, S., Ashokkumar, M. (2005). Effect of surfactants on the rate of growth of an air bubble by rectified diffusion. The Journal of Physical Chemistry B, 109 (30), 14595-14598.

[6] Lange, D.F., Bruin, G.J., Wijngaarden, L. (1994). On the mechanism of cloud cavitation - experiment and modeling. In Second International Symposium on Cavitation, April 5-7, 1994, Tokyo, Japan, 45-49.

[7] Reisman, G.E., Wang, Y.C., Brennen, C.E. (1998). Observations of shock waves in cloud cavitation. Journal of Fluid Mechanics, 355, 255-283.

[8] Zhang, J.M., Qing, Y.A.N.G., Wang, Y.R., Xu, W.L., Chen, J.G. (2011). Experimental investigation of cavitation in a sudden expansion pipe. Journal of Hydrodynamics, Ser. B, 23 (3), 348-352.

[9] Dvorsky, R., Lunacek, J., Sliva, A. (2011). Dynamics analysis of cavitation disintegration of microparticles during nanopowder preparation in a new Water Jet Mill (WJM) device. Advanced Powder Technology, 22 (5), 639-643.

[10] Olšiak, R., Knížat, B., Mlkvik, M. (2012). Visualization of cavitating micro jets. EPJ Web of Conferences, 25, 01062.

[11] Kirschner, O. (2005). Vortex rope measurement in a pump-turbine with a straight cone draft tube. In Workshop on Turbomachinery Hydromechanics, Timisoara, Romania, Vol. 23.
[12] Amirante, R., Distaso, E., Tamburrano, P. (2014). Experimental and numerical analysis of cavitation in hydraulic proportional directional valves. Energy Conversion and Management, 87, 208-219.

[13] Jančula, D., Mikula, P., Maršálek, B., Rudolf, P., Pochylý, F. (2014). Selective method for cyanobacterial bloom removal: Hydraulic jet cavitation experience. Aquaculture International, 22 (2), 509-521.

[14] Noskievič, J. (1969) Cavitation. Prague, Czech Republic: Academia. (in Czech)

[15] Yan, Z., Liu, J., Chen, B., Cheng, X., Yang, J. (2015). Fluid cavitation detection method with phase demodulation of ultrasonic signal. Applied Acoustics, 87, 198-204.

[16] Wójs, K. (2004) Kawitacja w cieczach o różnych właściwościach reologicznych. Wrocław, Poland: Oficyna Wydawnicza Politechniki Wrocławskiej.

[17] Kuneš, J. (2012). Dimensionless Physical Quantities in Science and Engineering. Elsevier.

[18] Lira, I., Grientschnig, D. (2013). A formalism for expressing the probability density functions of interrelated quantities. Measurement Science Review, 13 (2), 50-55.

[19] Shalnev, K.K. (1954). The structure of the cavitation region. Izvestiya Akademii Nauk SSR, 5, 119-146. (in Russian)

[20] Hunsaker, J.C. (1935) Cavitation research - a progress report on work at the Massachusetts Institute of Technology. Mechanical Engineering, April 1935, 211-216.

[21] Pearsall, I.S. (1966). Paper 14: Acoustic detection of cavitation. Proceedings of the Institution of Mechanical Engineers, Conference Proceedings, 181 (1), 1-8.

[22] Sou, A., Hosokawa, S., Tomiyama, A. (2007). Effects of cavitation in a nozzle on liquid jet atomization. International Journal of Heat and Mass Transfer, 50 (17), 3575-3582.

[23] Noskievič, J. (1987). Fluid Mechanics. Prague, Czech Republic: SNTL. (in Czech).

Received February 24, 2016. Accepted July 25, 2016. 